\title{
Minimally invasive pancreatic surgery-will robotic surgery be the future?
}

\author{
Luca Dittrich (D) - Matthias Biebl · Thomas Malinka · Michael Knoop · Johann Pratschke
}

Received: 28 November 2020 / Accepted: 16 December 2020 / Published online: 27 January 2021

(C) The Author(s) 2021

Summary Due to the complexity of the procedures and the texture of the organ itself, pancreatic surgery remains a challenge in the field of visceral surgery. During the past decade, a minimally invasive approach to pancreatic surgery has gained distribution in clinical routine, extending from left-sided procedures to pancreatic head resections. While a laparoscopic approach has proven beneficial for many patients with left-sided pancreatic pathologies, the complex reconstruction in pancreas head resections remains worrisome with the laparoscopic approach. The robotic technique was established to overcome such technical constraints while preserving the advantages of the laparoscopic approach. Even though robotic systems are still in development, especially in pancreatoduodenectomy, the current literature demonstrates the feasibility of this approach and stable clinical and oncological outcomes compared to the open technique, albeit only under the condition of such operations being performed by specialist teams in a high-volume setting ( $>20$ robotic pancreaticoduodenectomies per year). The aim of this review is to analyze the current evidence regarding a minimally invasive approach to pancreatic surgery and to review the potential of a robotic approach. Presently, there is still a scarcity of sound evidence and long-term oncological data regarding the role of minimally invasive and robotic pancreatic surgery in the literature, especially in the setting of pancreaticoduodenectomy.

\footnotetext{
L. Dittrich, MD $(\bowtie) \cdot$ Univ.-Prof. M. Biebl, MD •

T. Malinka, MD · M. Knoop, MD · Univ.-Prof. J. Pratschke, MD Department of Surgery, Campus Charité Mitte and Campus Virchow-Klinikum, Charité-Universitätsmedizin, Corporate Member of Freie Universität Berlin, Humboldt-Universität zu Berlin, Augustenburger Platz 1, 13353 Berlin, Germany luca.dittrich@charite.de
}

Keywords Robot-assisted surgery • Pancreatoduodenectomy · Distal pancreatectomy • Cost-effectiveness $\cdot$ Review

\section{Introduction}

Pancreatic surgery is among the most challenging procedures in visceral surgery and burdened with high morbidity and mortality rates $[1,2]$.

Minimally invasive pancreatic surgery was first introduced in 1994 by Gagner and Pomp [3] and conveys the advantages of a laparoscopic approach, such as reduced operative blood loss, shorter length of stay, and reduced surgical site infections. Especially in the past decade, minimally invasive pancreatic surgery became more widespread [4-9]. This mainly relates to laparoscopic distal pancreatectomy (LDP), whereas laparoscopic pancreaticoduodenectomy (LPD) is lacking popularity among most pancreas surgeons [10, 11]. One of the main reasons for the rather slow uptake of LPD is the complexity of the procedure itself. Especially the reconstruction with three anastomoses in a laparoscopic setting with two-dimensional imaging, the limited degree of freedom of movement, and the fulcrum effect remains challenging $[12,13]$.

To overcome the previously described disadvantages of the laparoscopic approach, robotic surgical systems have been introduced [14]. By providing a three-dimensional stereoscopic view, a reduction of tremor, and seven degrees of freedom, robotic systems support the surgeon in intracorporal suturing and dissection, whilst offering ergonomic comfort. The currently most commonly used robotic platform is the DaVinci ${ }^{\circledR}$ surgical system (Intuitive Surgical ${ }^{\circledR}$ Inc., Sunnyvale, CA, USA).

In 2003, the first case series of robot-assisted pancreaticoduodenectomy was published by Giulianotti et al., suggesting that robotic pancreas surgery is safe 
and feasible in a clinical setting [15]. Since then, several series of robotic pancreas surgery have been published with a wide range of indications (pancreatoduodenectomy, distal pancreatectomy, total pancreatectomy, pancreatic tumor enucleation, etc.) [16, 17]. Despite numerous promising results in robotic surgery of the pancreas, relevant controversies like cost-effectiveness still remain [18, 19].

Nevertheless, trend analyses from the US and Europe demonstrate a steady increase in usage of robotic pancreas surgery. In 2018, Stewart et al. reported an overall fivefold increase $(<1 \%$ to $3 \%)$ in 4 years in robotic surgery using data from a national US database. In the same time period, an increase in laparoscopic surgeries from $10 \%$ to $13 \%$ and a decrease in open surgery from $89 \%$ to $84 \%$ were reported $(p<0.001)$ [20]. In 2014, European data demonstrated that robot-assisted minimally invasive pancreatic surgery was performed by $14 \%$ of the surveyed surgeons [8].

\section{Pancreatic surgery}

The main pancreatic procedures are a pancreatoduodenectomy (PD) and a distal pancreatectomy (DP). Main indications for DP include benign pathologies, premalignant (e.g., IPMN), and malignant tumors (e.g., pancreatic ductal adenocarcinoma, pancreatic neuroendocrine, etc.) [21-23].

The vast majority of the indications for PD are malignant tumors located in the periampullary region and the head of the pancreas. Only a small percentage of the performed procedures are because of nonmalignant neoplastic diseases and chronic pancreatitis [24-26].

Despite a continuous improvement in surgical technique and postoperative care, mortality rates are still high [1, 2]. Recent nationwide data from Germany report an overall in-hospital mortality of $10.1 \%$ for pancreatic surgery. Mortality rate was highest in total pancreatectomy compared to PD and DP $(22.9 \%$, $7.7 \%$, and $7.3 \%$, respectively) [27]. In 2019, a retrospective analysis reported an in-hospital mortality rate of almost $7 \%$ and a severe complication rate (like sepsis, peritonitis, ventilation $>48 \mathrm{~h}$ ) of up to $27.7 \%$ in approximately 70,000 patients who underwent pancreatic surgery, making it one of the most complex procedures in visceral surgery [28]. One of the major factors influencing morbidity and mortality rates is the postoperative development of pancreatic surgeryspecific complications like postoperative pancreatic fistula (POPF) and post-pancreatectomy hemorrhage $(\mathrm{PPH})$. POPF grades $\mathrm{B}$ and $\mathrm{C}$ are considered to be clinically relevant (CR-POPF) according to the International Study Group of Pancreatic Fistula (ISGPF) [29]. CR-POPF rates have been shown to be between 20 and $40 \%$ in recent literature $[23,30,31]$.

\section{Laparoscopic pancreatic surgery}

Patients after laparoscopic pancreatic surgery benefit from shorter length of hospital stay, reduced operative blood loss, and less delayed gastric emptying (DGE) $[6,7,23,32]$. However, particularly in laparoscopic $\mathrm{PD}$, concerns regarding safety were raised. Increased rates of postoperative bleeding, CR-POPF, and higher readmission rates were observed [33-35].

\section{Laparoscopic distal pancreatectomy}

Laparoscopic distal pancreatectomy (LDP), first described in 1994, has been shown to be much more applicable than LPD due to the absence of challenging reconstructions [10, 36-38].

In a multicenter randomized controlled trial (LEOPARD) comparing minimally invasive DP (MIDP) vs. open DP (ODP), the authors reported MIDP to be associated with a statistically significant decrease in intraoperative blood loss, time to functional recovery, length of hospital stay, and DGE. Further, the overall complication rate did not differ from ODP. Additionally, a conversion rate from MIDP to open DP of $8 \%$ was observed [23]. Further reports as well as consensus statements displayed superiority of LDP over ODP, while reporting non-inferiority in terms of surgical radicality ( $\mathrm{R} 0$ resection, lymph nodes harvested) [32, 39]. Therefore, several authors stated that MIDP is the treatment of choice for patients with left-sided pancreatic tumors [23, 32, 39].

In conclusion, laparoscopic distal pancreatectomy is recognized as a well-established approach to leftsided resections for benign as well as confined malignant lesions. Within these borders, it may be considered the treatment of first choice in patients with lesions left of the portomesenteric axis without vascular involvement.

\section{Laparoscopic pancreatoduodenectomy}

Laparoscopic PD is technically more advanced than LDP due to the complexity of the dissection and the following reconstruction. To compare the safety and feasibility of the laparoscopic approach, large observational studies were initiated. Recent randomized clinical trials have shown similar or lower postoperative morbidity and mortality rates (including POPF and DGE) as well as a shortened length of hospital stay after laparoscopic vs. open PD [40, 41]. Controversially, serious doubts regarding the safety of the laparoscopic approach were raised by various study groups. Dokmak et al. reported statistically significantly higher rates of severe morbidity $(28 \%$ vs. $20 \%, p=0.32$ ) in LPD compared to OPD due to grade C POPF (24\% vs. $6 \%, p=0.007)$, postoperative bleeding (24\% vs. $7 \%, p=0.02)$, and revision surgery ( $24 \%$ vs $11 \%, p=0.09$ ) [33]. A Europe-wide propensity score-matched study not only observed higher 
CR-POPF rates in LPD vs. OPD $(22.7 \%$ vs. $12.7 \%$, $p<0.001$ ), but also a prolonged length of hospital stay in the LPD group $(p<0.001)$ [42]. It is worth noting that a multicenter, patient-blinded, randomized controlled phase $2 / 3$ trial comparing LPD with OPD (LEOPARD-2) was prematurely terminated because of safety concerns related to higher 90-day complication-related mortality in the laparoscopic group $(10 \%$ vs. $2 \%, p=0.2$ ) [43].

In conclusion, for LPD, relevant safety concerns prevail and result in the statement that laparoscopic PD should not be part of the clinical routine outside expert centers.

\section{Robotic pancreatic surgery}

The robotic approach is supposed to overcome the difficulties of the laparoscopic technique in pancreatic surgery. Because of the stable $3 \mathrm{D}$ vision, a reduction of tremor, and the wristed instruments, the robotic system allows for easier resection and particularly supports the surgeon in the demanding process of reconstruction. Of note, unlike open or laparoscopic surgery, the robotic technique is still evolving through ongoing development of robotic systems.

\section{Robotic distal pancreatectomy}

Minimally invasive distal pancreatectomy is often adopted first due to the absence of the requirement of a complex reconstruction. As mentioned above, the laparoscopic approach is safe and feasible in DP and is considered as a primary treatment for leftsided pancreatic tumors [23].

Zhao et al. showed a lower blood transfusion rate, lower complication rate, and shorter length of hospital stay for RDP compared to OPD, while no difference was detected in POPF and mortality rates [16].

Data comparing robotic DP (RDP) and LDP seem to differ along current studies. The most reported advantage of RDP is a significantly lower conversion rate [22, 44-46]. Daouadi et al. not only reported a lower conversion rate $(0 \%$ vs. $16 \%, p<0.05)$ but also shorter operative time $(p<0.01)$ and lower median estimated blood loss $(p<0.05)$ for the RDP group [47]. The vast majority of recent data show comparable results in overall survival, oncological outcomes (negative resection margin, harvested lymph nodes), and postoperative complication rates (POPF, etc.) between RDP and LDP $[44,46,48]$.

Data on operative time are controversial in different trials and are reported to be comparable, in favor of LDP over RDP or the other way round [47-50], and mainly seem to be related to patient selection in the respective groups. Comparing all available techniques, Magge et al. showed the lowest operative time in RDP compared to the laparoscopic and open approach $(211 \pm 68 \mathrm{~min}, 318 \pm 124 \mathrm{~min}, 316 \pm 140 \mathrm{~min}$, respectively, $p<0.0001$ ) [22].
Depending on the indication, DP requires splenectomy. Spleen resection is associated with increased postoperative infections, overall complications, and long-term cardiovascular complications [49, 50]. Therefore, if the indication for splenectomy in DP is not given, especially in benign and low-grade pancreatic tumors, a spleen-preserving DP should be performed [50]. Numerous studies have shown a significant increase in spleen-preserving rates in RDP compared to LDP and ODP [51-53]. The randomized controlled LEOPARD trial reported spleen-preserving procedures in $45 \%$ of all MIDP (42 LDP, 5 RDP) and $50 \%$ in ODP $(p=0.61)$. In contrast, a single-surgeon experience from 2013 performed spleen-preserving RDP in $95.5 \%[23,54]$.

Regarding measurement of the surgical learning curve, one of the most reported aspects is operative time. The operative time in RDP seems to drop significantly after the first 20 cases compared to initial operative time $(p<0.0001)$ and the readmission rate was reduced significantly after 40 procedures $(p=0.04)$, leading to the conclusion that RDP outcomes were optimized after 40 cases [55]. In another study, the operative time was even reduced significantly after 10 performed robotic DPs [56].

In conclusion, robotic DP appears to lead to a reduced conversion to open resection rate and an increase of spleen-preserving procedures in patients with non-carcinomatous lesions, due to superior vascular control compared to the laparoscopic approach. Morbidity rates and oncological outcomes appear to be equal to LDP.

\section{Robotic pancreatoduodenectomy}

An early study from 2003 by Giulianotti et al. analyzed eight pancreatoduodenectomies, six procedures in hybrid technique (laparoscopy for resection and robotic reconstruction), and two fully robotic techniques, with a morbidity rate of $37.5 \%$ and mortality rate of $12.5 \%$ [15]. Ten years later, Giulianotti published a single-surgeon experience with 134 patients of robot-assisted surgery for various pancreatic diseases. The majority of the procedures were PD ( $n=60)$, followed by 46 DP (13 spleen-preserving DP and 13 splenopancreatectomies). The reported overall morbidity rate in this study was $26.0 \%$ and the mortality rate was $2.23 \%$ [57].

A retrospective review of a prospective database by Zureikat et al. in 2013 showed a 30-day and 90day mortality rate of $0.8 \%$ and $2.0 \%$, respectively, in 250 robotic pancreatic resections. Complication rates, including the Clavien-Dindo classifications 3 and 4, were $14 \%$ and $6 \%$, respectively, and the conversion rate to open surgery was $6 \%$ [21].

Various studies show that RPD is associated with less blood loss, while the length of hospital stay is controversial. Mortality rate and overall postoperative complication rate including pancreatic fistula (POPF) 
of RPD have been shown to be comparable to OPD, whereas operative time is reported to be longer in RPD. In 2018, Wang et al. compared RPD with OPD ( $n=87$ each), showing a significantly lower median blood loss in the RPD vs. the OPD group $(120 \mathrm{ml}$ vs. $250 \mathrm{ml}, p=0.001)$. Meanwhile, the operative time was longer in the RPD group ( $420 \mathrm{~min}$ vs. $360 \mathrm{~min}$, $p<0.001$ ) [26]. In a single-center study by Lei et al., 20 RPD were compared with 67 OPD. They observed a longer operative time (mean 491.5 vs. $264.9 \mathrm{~min}$ ) and reduced blood loss (mean 247 vs. $774.8 \mathrm{ml}$ ) for RPD [58]. In a meta-analysis from 2020 including 18 nonrandomized prospective and retrospective studies comparing RPD $(n=1593)$ and OPD $(n=12,046)$, the reported estimated intraoperative blood loss was significantly lower in the RDP group $(352.1 \pm 174.1$ vs. $588.4 \pm 219.4 ; p=0.0003)$. However, operative time was longer in RDP than in OPD (461.1 \pm 84 vs. $384.2 \pm 73.8$; $p=0.0004$ ) [31]. In contrast, a study group from Great Britain could not find any discrepancy in estimated blood loss or operative time between RPD and LPD [59].

Similar to distal pancreatectomy, several studies noticed lower conversion rates to open surgery for RPD compared to LPD [59-61].

The length of hospital stay is controversial and reported to be significantly shorter in RPD than in OPD and even laparoscopic PD (LPD), whereas other studies reported equal times between RPD and OPD [16, $20,31,58,59,62,63]$.

Kowalsky et al. demonstrated that postoperative complications and postoperative intensive care unit (ICU) admissions were significantly lower in patients with robotic surgery than in open surgery. Major complications (Clavien-Dindo $>2, \quad p=0.241$ ), CR-POPF $(p=0.061)$, and DGE $(p=0.268)$ were similar in both cohorts [63].

A meta-analysis from 2020 did not find significant differences in mortality, morbidity, grade B POPF, grade C POPF, overall POPF, and DGE between RPD $(n=1593)$ and OPD $(n=12,046)$ [31]. Another metaanalysis revealed significant advantages in the RPD group regarding overall complications $(p=0.02)$ and wound infections $(p=0.01)$, while POPF did not vary between RPD $(n=809)$ and OPD $(n=2881)$ [16].

In a recent meta-analysis, the comparison between RPD $(n=1025)$ and LPD $(n=2437)$ did not show differences in overall postoperative complication rates or the incidence of POPF [59], and single-center data from the University of Pittsburgh comparing patients undergoing RPD and OPD reported overall POPF rates to be similar in both groups, but RPD to be associated with a significantly lower rate of CR-POPF $(6.7 \%$ vs. $15.8 \%, p<0.001$ ) [30].

Most indications for PDs are due to malignant disease. Therefore, the oncological outcome is essential for the implementation of the robotic approach in pancreatic surgery. Since robust oncological data are not available in the early evaluation phase of new sur- gical techniques, oncological outcome is extrapolated through perioperative surrogate parameters such as surgical resection margin ( $\mathrm{R}$ status) and number of harvested lymph nodes. The majority of current studies and meta-analysis report RPD to be associated with a higher R0 resection rate and no significant difference in harvested lymph nodes compared to OPD $[16,64]$.

A propensity score-matched study by Wang et al. reported similar R0 resection rates between RPD $(n=87)$ and OPD $(n=87)$, while the number of harvested lymph nodes was significantly higher in the RPD group. The 1-, 2-, and 3-year overall survival rates were significantly higher in the RPD than in the OPD group $(89.9 \%, 96.2 \%, 93.2 \%$ and $95.5 \%, 90.0 \%$, $85.2 \%$, respectively, $p=0.009$ ) before propensity score matching (PSM). After PSM, no significant difference was found between the two groups [26].

A study by Nassour et al. compared RPD and LPD and reported equal rates of negative resection margins and numbers of harvested lymph nodes in both groups [60].

Podda et al. concluded in their meta-analysis that RPD ( $n=1593)$ is reliable regarding the oncological outcome, with no difference in surgical radicality as reported by positive resection margin status $(13.3 \%$ vs $16.1 \% ; P=0.32)$ and number of harvested lymph nodes $(19.1 \pm 9.9$ vs $17.3 \pm 9.9 ; P=0.22)$ compared to OPD $(n=12,046)$ [31].

In 2019, the Pittsburgh group reported a significantly longer median overall survival in the robotic cohort in a retrospective review of 456 pancreatectomies (226 robotic vs. 230 open procedures with a 24 -month follow-up). Consequently, the authors concluded that robotic pancreatic resections are not inferior to open pancreatic resections regarding oncological outcome [65].

Even though promising results of RPD exist, the complexity of the procedure should not be underestimated. The Miami guidelines on minimally invasive pancreas resection recommend an annual volume of 20 RPDs per center per year due to a reduced complication and mortality rate in centers with $>20$ RPDs and $>10$ RPDs per year, respectively [66].

The learning curve for LPD differs between 10 and 50 cases depending on the chosen outcome parameter (e.g., operative time, complication rate, etc.) [67-69]. In robotic surgery, a single-surgeon series reported a decrease in operative time after approximately 15 performed RPDs, a decrease in overall complication rate was seen after 15 cases, whereas major complications (Clavien-Dindo 3-5) decreased after 30 cases [70].

Boone et al. showed a statistically significant improvement in blood loss and conversion to open surgery rates after 20 cases of RPD, a reduction in the incidence of pancreatic fistulas after 40 cases, and a significant reduction in operative times after 80 cases [71]. 
Another retrospective study analyzed the learning curve of a single surgeon after 100 RPDs. The mean operation time, length of hospital stay, and estimated blood loss were significantly decreased after 40 cases $(p<0.05)$. POPF and DGE rates decreased after 60 cases $(p<0.05)$ [72].

A retrospective study from China observed significant improvements in operative and oncological outcomes after 250 cases [73].

Jones et al. resume in a review published in 2020 that RPD can be performed safely and feasibly when specifically trained surgeons, who have undergone a proficiency-based training program, can maintain a minimum of 20 RPD procedures per year. Considering that even in highly trained centers only a maximum of $40-50 \%$ of patients requiring PD will be eligible for RPD, at least 50 PDs annually should be performed to fulfill the criteria of 20 RPDs per year [74].

To conclude, RPD seems to be a promising alternative when performed by highly experienced surgeons in a high-volume center.

\section{Procedural costs and cost-effectiveness of robotic PD and DP}

Both procedures are associated with a major debate of economics. Additional costs of robotic surgery cannot be ignored. However, data on the cost-effectiveness of robotic surgery vary compared to the laparoscopic and the open approach [22, 46, 48, 75, 76]. Souche et al. report that short-term results of RDP seem to be equal to LDP; nevertheless, the significantly higher costs make RDP a non-cost-effective approach [18]. Cost analysis performed by Higgins et al. found a significant increase in costs for robotic surgery caused by consumable surgical supplies of the robotic platform [77]. A PSM study analyzed intraoperative and overall costs for hospitalization for robotic DP and LDP. Higher costs in both subgroups for RDP were demonstrated $(p<0.001)$, whereas no difference was found in the analysis of postoperative costs between these two surgical approaches $(p=0.649)$ [19]. Interestingly, a study reported a significant overall cost decrease in patients who underwent RPD after implantation of the "enhanced recovery after surgery" (ERAS) pathway compared to RPD (pre-ERAS) and OPD (pre-ERAS and ERAS) [63].

\section{Discussion and conclusion}

Robotic surgery is a promising approach for pancreatic resections. The current literature demonstrates that robotic PD and DP are safe and feasible when compared to open and laparoscopic techniques.

In terms of intraoperative data, the majority of studies show longer operative time but less blood loss in robotic surgery compared to the open approach. Additionally, aside from conversion to open surgery rates [22, 44, 60, 61], no widespread disparity is reported between the robotic and laparoscopic approaches [16, 46, 59]. Despite one high-volume center study with significantly reduced incidence of CR-POPF in RPD, there are no decisive data to identify differences of either minimally invasive approach (robotic/laparoscopic) or open surgery regarding PDspecific complications like POPF and DGE [16, 30, 59, 63]. In contrast, the overall complication rate seems to be significantly lower in robotic PD and DP compared to open PD an DP, with no difference between robotic and laparoscopic pancreatic surgery $[16,48$, $59,63]$.

An additional advantage of RDP over LDP is the significantly higher spleen-preserving rate in RDP [51, 53].

Major concerns which have to be eliminated before using robotic surgery in daily clinical routine are the lacking oncological long-term data. The essential basis is formed by the encouraging data on shortand mid-term oncological results. The number of harvested lymph nodes and negative resection margins (R0) are comparable in RDP and LDP, which is accepted as a safe and effective surgical approach for distal pancreatectomy [44, 46]. Similar results were shown in the first studies comparing OPD and RPD. Girgis et al. showed that RPD is an independent factor for improved survival rates (HR 0.75; $p=0.05$ ) [65]. Negative resection margin and number of harvested lymph nodes seem to be equal between OPD and RPD, or even favor RPD [31, 64]. Nevertheless, these results must be interpreted with caution. A treatment allocation bias might exist in robotic pancreatic resections, since fewer patients received neoadjuvant chemo- or radiotherapy and tumor size was significantly smaller in robotic surgery [39, 78].

Cost-effectiveness is still a controversial topic in robotic surgery. The majority of the presented data regarding cost-effectiveness in robotic DP report higher overall costs, even though some data are in favor of robotic procedures. To date, the market size for robotic surgery is limited and held as a monopoly by Intuitive Surgical ${ }^{\circledR}$ Inc. (Sunnyvale, CA, USA). Nevertheless, even a small market size may lead to entering of different companies and consecutively to reduced costs for robotic surgical technology due to more competition between companies [79, 80].

Taking the current literature into consideration, robotic pancreatic surgery seems to be a feasible and safe alternative to the well-known open and laparoscopic approaches, while expanding continuously. Especially in PD, robotic surgery could be shown to improve clinical and oncological outcomes, although procedural costs are still high. Even for distal pancreatectomy, the robotic approach may enrich daily clinical routine, despite missing major improvements and higher costs compared to LDP, due to a significantly lower conversion rate and higher spleenpreserving rate. 
More RCTs and long-term oncological data are needed to overcome the remaining obstacles for robotic PD and DP. The current literature is promising and may lead robotics to a change in the current praxis of pancreatic surgery, to become standard in surgical routine.

Author contribution The invitation to Prof. Pratschke J on behalf of the editors of European Surgery-Acta Chirurgica Austriaca (ES ACA) led to this review article. The authors and co-authors Dittrich L, Biebl M, and Malinka T performed the literature search on the topic and wrote the article. Proof reading and critical revision of the article was performed by Biebl M, Malinka T, Knoop M, and Pratschke J. Final approval was given by Prof. Pratschke.

Funding Open Access funding enabled and organized by Projekt DEAL.

Conflict of interest L. Dittrich, M. Biebl, T. Malinka, M. Knoop, and J. Pratschke declare that they have no competing interests.

Open Access This article is licensed under a Creative Commons Attribution 4.0 International License, which permits use, sharing, adaptation, distribution and reproduction in any medium or format, as long as you give appropriate credit to the original author(s) and the source, provide a link to the Creative Commons licence, and indicate if changes were made. The images or other third party material in this article are included in the article's Creative Commons licence, unless indicated otherwise in a credit line to the material. If material is not included in the article's Creative Commons licence and your intended use is not permitted by statutory regulation or exceeds the permitted use, you will need to obtain permission directly from the copyright holder. To view a copy of this licence, visit http://creativecommons.org/licenses/by/4.0/.

\section{References}

1. HeJ,AhujaN, MakaryMA, etal. 2564 resected periampullary adenocarcinomas at a single institution: trends over three decades. HPB. 2014;16(1):83-90.

2. Winter JM, Cameron JL, Campbell KA, et al. 1423 pancreaticoduodenectomies for pancreatic cancer: a single-institution experience. J Gastrointest Surg. 2006;10(9):1191-9.

3. Gagner M, Pomp A. Laparoscopic pylorus-preserving pancreatoduodenectomy. Surg Endosc. 1994;8(5):408-10.

4. Morneau M, Boulanger J, Charlebois P, et al. Laparoscopic versus open surgery for the treatment of colorectal cancer: a literature review and recommendations from the Comité de l'évolution des pratiques en oncologie. Can J Surg. 2013;56(5):297-310.

5. Bonjer HJ, Deijen CL, Abis GA, et al. A randomized trial of laparoscopic versus open surgery for rectal cancer. N Engl J Med. 2015;372(14):1324-32.

6. Kang S-B, ParkJW, Jeong S-Y, etal. Open versuslaparoscopic surgery for mid or low rectal cancer after neoadjuvant chemoradiotherapy (COREAN trial): short-term outcomes of an open-label randomised controlled trial. Lancet Oncol. 2010;11(7):637-45.

7. KendrickML, Cusati D. Total laparoscopic pancreaticoduodenectomy: feasibility and outcome in an early experience. Arch Surg. 2010;145(1):19-23.

8. de Rooij T, Besselink MG, Shamali A, et al. Pan-European survey on the implementation of minimally inva- sive pancreatic surgery with emphasis on cancer. HPB. 2016;18(2):170-6.

9. van Hilst J, de Rooij T, Abu Hilal M, et al. Worldwide survey on opinions and use of minimally invasive pancreatic resection. HPB. 2017;19(3):190-204.

10. Kendrick ML. Laparoscopic and robotic resection for pancreatic cancer. Cancer J.2012;18(6):571-6.

11. Rosales-Velderrain A, Bowers SP, Goldberg RF, et al. National trends in resection of the distal pancreas. World J Gastroenterol. 2012;18(32):4342-9.

12. Heemskerk J, Zandbergen R, Maessen JG, et al. Advantages of advanced laparoscopic systems. Surg Endosc. 2006;20(5):730-3.

13. Liang S, Hameed U, Jayaraman S. Laparoscopic pancreatectomy: indications and outcomes. World J Gastroenterol. 2014;20(39):14246-54.

14. Lanfranco AR, Castellanos AE, Desai JP, et al. Robotic surgery: a current perspective. AnnSurg. 2004;239(1):14-21.

15. Giulianotti PC, Coratti A, Angelini M, et al. Robotics in general surgery: personal experience in a large community hospital. Arch Surg. 2003;138(7):777-84.

16. Zhao W, Liu C, Li S, et al. Safety and efficacy for robotassisted versus open pancreaticoduodenectomy and distal pancreatectomy: a systematic review and meta-analysis. Surg Oncol. 2018;27(3):468-78.

17. Liu R, Zhao G-D, Tang W-B, et al. A single-team experience with robotic pancreatic surgery in 1010 cases. Nan Fang Yi KeDaXueXue Bao. 2018;38(2):130-4.

18. Souche R, Herrero A, Bourel G, et al. Robotic versus laparoscopic distal pancreatectomy: a French prospective single-center experience and cost-effectiveness analysis. SurgEndosc. 2018;32(8):3562-9.

19. De Pastena M, Esposito A, Paiella S, et al. Cost-effectiveness and quality oflife analysis oflaparoscopic and robotic distal pancreatectomy: a propensity score-matched study. Surg Endosc. 2020; https://doi.org/10.1007/s00464-020-075281 .

20. StewartCL, ItuartePHG, MelstromKA, etal. Robotic surgery trends in general surgical oncology from the National InpatientSample. Surg Endosc. 2019;33(8):2591-601.

21. Zureikat AH, Moser AJ, Boone BA, et al. 250 robotic pancreatic resections: safety and feasibility. Ann Surg. 2013;258(4):554-62.

22. MaggeDR, Zenati MS, HamadA, etal. Comprehensive comparative analysis of cost-effectiveness and perioperative outcomes between open, laparoscopic, and robotic distal pancreatectomy. HPB. 2018;20(12):1172-80.

23. de Rooij T, van Hilst J, van Santvoort H, et al. Minimally invasive versus open distal pancreatectomy (LEOPARD): a multicenter patient-blinded randomized controlled trial. Ann Surg. 2019;269(1):2-9.

24. Napoli N, Kauffmann EF, Menonna F, et al. Indications, technique, and results of robotic pancreatoduodenectomy. Updates Surg. 2016;68(3):295-305.

25. Rosemurgy A, Ross S, Bourdeau T, et al. Robotic pancreaticoduodenectomy is the future: here and now. J Am Coll Surg. 2019;228(4):613-24.

26. Wang S-E, Shyr B-U, Chen S-C, et al. Comparison between robotic and open pancreaticoduodenectomy with modified Blumgart pancreaticojejunostomy: a propensity scorematched study. Surgery. 2018;164(6):1162-7.

27. Nimptsch U, Krautz C, Weber GF, et al. Nationwide inhospital mortality following pancreatic surgery in Germany is higher than anticipated. Ann Surg. 2016;264(6):1082-90.

28. Baum P, Diers J, Lichthardt S, et al. Mortality and complications following visceral surgery: a nationwide analysis 
based on the diagnostic categories used in German hospital invoicing data. Dtsch Arztebl Int. 2019;116(44):739-46.

29. Bassi C, Marchegiani G, Dervenis C, et al. The 2016 update of the International Study Group (ISGPS) definition and grading of postoperative pancreatic fistula: 11 years after. Surgery. 2017;161(3):584-91.

30. Cai J, Ramanathan R, Zenati MS, et al. Robotic pancreaticoduodenectomy is associated with decreased clinically relevant pancreatic fistulas: a propensity-matched analysis. J Gastrointest Surg. 2020;24(5):1111-8.

31. Podda M, Gerardi C, Di Saverio S, et al. Robotic-assisted versus open pancreaticoduodenectomy for patients with benign and malignant periampullary disease: a systematic review and meta-analysis of short-term outcomes. Surg Endosc. 2020;34(6):2390-409.

32. Edwin B, Sahakyan MA, Abu Hilal M, et al. Laparoscopic surgery for pancreatic neoplasms: the European association for endoscopic surgery clinical consensus conference. SurgEndosc. 2017;31(5):2023-41.

33. Dokmak S, Ftériche FS, Aussilhou B, et al. Laparoscopic pancreaticoduodenectomy should not be routine for resection of periampullary tumors. J Am Coll Surg. 2015;220(5):831-8.

34. Nassour I, Wang SC, Christie A, et al. Minimally invasive versus open pancreaticoduodenectomy: a propensitymatched study from a national cohort of patients. Ann Surg. 2018;268(1):151-7.

35. Chopinet S, Fuks D, Rinaudo M, et al. Postoperative bleeding after laparoscopic pancreaticoduodenectomy: the Achilles' heel? World J Surg. 2018;42(4):1138-46.

36. Cuschieri A. Laparoscopic surgery of the pancreas. J R Coll Surg Edinb. 1994;39(3):178-84.

37. Cuschieri A, Jakimowicz JJ, van Spreeuwel J. Laparoscopic distal $70 \%$ pancreatectomy and splenectomy for chronic pancreatitis. Ann Surg. 1996;223(3):280-5.

38. MabrutJ-Y,Fernandez-CruzL,AzagraJS, etal. Laparoscopic pancreatic resection: results of a multicenter European study of 127 patients. Surgery. 2005;137(6):597-605.

39. Ricci C, Casadei R, Taffurelli G, et al. Laparoscopic versus open distal pancreatectomy for ductal adenocarcinoma: a systematic review and meta-analysis. J Gastrointest Surg. 2015;19(4):770-81.

40. Palanivelu C, Senthilnathan P, Sabnis SC, et al. Randomized clinical trial of laparoscopic versus open pancreatoduodenectomy for periampullary tumours. Br J Surg. 2017;104(11):1443-50.

41. Poves I, Burdío F, Morató O, et al. Comparison of perioperative outcomes between laparoscopic and open approach for pancreatoduodenectomy: the PADULAP randomized controlled trial. Ann Surg. 2018;268(5):731-9.

42. Klompmaker S, van Hilst J, Wellner UF, et al. Outcomes after minimally-invasive versus open pancreatoduodenectomy: a pan-European propensity score matched study. AnnSurg. 2020;271(2):356-63.

43. van Hilst J, de Rooij T, Bosscha K, et al. Laparoscopic versus open pancreatoduodenectomy for pancreatic or periampullary tumours (LEOPARD-2): a multicentre, patientblinded, randomised controlled phase 2/3 trial. Lancet Gastroenterol Hepatol. 2019;4(3):199-207.

44. Raoof M, Nota CLMA, Melstrom LG, et al. Oncologic outcomes after robot-assisted versus laparoscopic distal pancreatectomy: analysis of the National Cancer Database. J Surg Oncol. 2018;118(4):651-6.

45. Kamarajah SK, Sutandi N, Robinson SR, et al. Robotic versus conventional laparoscopic distal pancreatic resection: a systematic review and meta-analysis. HPB. 2019;21(9):1107-18.
46. Xu S-B, Jia C-K, Wang J-R, et al. Do patients benefit more from robot assisted approach than conventional laparoscopic distal pancreatectomy? A meta-analysis of perioperative and economic outcomes. J Formos Med Assoc. 2019;118(1 Pt2):268-78.

47. Daouadi M, Zureikat AH, Zenati MS, et al. Robot-assisted minimally invasive distal pancreatectomy is superior to the laparoscopic technique. Ann Surg. 2013;257(1):128-32.

48. LymanWB, Passeri M, SastryA, etal. Robotic-assisted versus laparoscopic left pancreatectomy at a high-volume, minimally invasive center. Surg Endosc. 2019;33(9):2991-3000.

49. Weledji EP. Benefits and risks of splenectomy. Int J Surg. 2014;12(2):113-9.

50. Shoup M, Brennan MF, McWhite K, et al. The value of splenic preservation with distal pancreatectomy. Arch Surg. 2002;137(2):164-8.

51. Waters JA, Canal DF, Wiebke EA, et al. Robotic distal pancreatectomy: cost effective? Surgery. 2010;148(4):814-23.

52. Hu Y-H, Qin Y-F, Yu D-D, et al. Meta-analysis of shortterm outcomes comparingrobot-assisted andlaparoscopic distal pancreatectomy. JComp Eff Res. 2020;9(3):201-18.

53. Goh BKP, Chan CY, Soh H-L, et al. A comparison between robotic-assisted laparoscopic distal pancreatectomy versus laparoscopic distal pancreatectomy. Int J Med Robot. 2017; https://doi.org/10.1002/rcs.1733.

54. Hwang HK, Kang CM, Chung YE, et al. Robot-assisted spleen-preserving distal pancreatectomy: a single surgeon's experiences and proposal of clinical application. SurgEndosc. 2013;27(3):774-81.

55. Shakir M, Boone BA, Polanco PM, et al. The learning curve for robotic distal pancreatectomy: an analysis of outcomes of the first 100 consecutive cases at a highvolume pancreatic centre. HPB. 2015;17(7):580-6.

56. Napoli N, Kauffmann EF, Perrone VG, et al. The learning curve in robotic distal pancreatectomy. Updates Surg. 2015;67(3):257-64.

57. Giulianotti PC, Sbrana F, Bianco FM, etal. Robot-assistedlaparoscopic pancreatic surgery: single-surgeon experience. SurgEndosc. 2010;24(7):1646-57.

58. Lai ECH, Yang GPC, Tang CN. Robot-assisted laparoscopic pancreaticoduodenectomy versus open pancreaticoduodenectomy - a comparative study. Int J Surg. 2012;10(9):475-9.

59. Kamarajah SK, Bundred J, Saint MO, et al. Robotic versus conventional laparoscopic pancreaticoduodenectomy a systematic review and meta-analysis. Eur J Surg Oncol. 2020;46(1):6-14.

60. Nassour I, Choti MA, Porembka MR, et al. Robotic-assisted versus laparoscopic pancreaticoduodenectomy: oncological outcomes. Surg Endosc. 2018;32(6):2907-13.

61. Zimmerman AM, Roye DG, Charpentier KP. A comparison of outcomes between open, laparoscopic and robotic pancreaticoduodenectomy. HPB. 2018;20(4):364-9.

62. McMillan MT, Zureikat AH, Hogg ME, et al. A propensity score-matched analysis of robotic vs open pancreatoduodenectomy on incidence of pancreatic fistula. JAMA Surg. 2017;152(4):327-35.

63. Kowalsky SJ, Zenati MS, Steve J, et al. A combination of robotic approach and ERAS pathway optimizes outcomes and cost for pancreatoduodenectomy. Ann Surg. 2019;269(6):1138-45.

64. Peng L, LinS, LiY, etal. Systematic review and meta-analysis of robotic versus open pancreaticoduodenectomy. Surg Endosc. 2017;31(8):3085-97.

65. Girgis MD, Zenati MS, King JC, et al. Oncologic outcomes after robotic pancreatic resections are not inferior to open 
surgery. Ann Surg. 2019; https://doi.org/10.1097/SLA. 0000000000003615.

66. Asbun HJ, Moekotte AL, Vissers FL, et al. The Miami international evidence-based guidelines on minimally invasive pancreas resection. Ann Surg. 2020;271(1):1-14.

67. Speicher PJ, Nussbaum DP, White RR, et al. Defining the learning curve for team-based laparoscopic pancreaticoduodenectomy. Ann Surg Oncol. 2014;21(12):4014-9.

68. Nagakawa Y, Nakamura Y, Honda G, et al. Learning curve and surgical factors influencing the surgical outcomes during the initial experience with laparoscopic pancreaticoduodenectomy. J Hepatobiliary Pancreat Sci. 2018;25(11):498-507.

69. WangM, MengL, CaiY, etal. Learning curveforlaparoscopic pancreaticoduodenectomy: a CUSUM analysis. J Gastrointest Surg. 2016;20(5):924-35.

70. Takahashi C, Shridhar R, Huston J, et al. Outcomes associated with robotic approach to pancreatic resections. J Gastrointest Oncol. 2018;9(5):936-41.

71. Boone BA, Zenati M, Hogg ME, et al. Assessment of quality outcomes for robotic pancreaticoduodenectomy: identification of the learning curve. JAMA Surg. 2015;150(5):416-22.

72. Zhang T, Zhao Z-M, Gao Y-X, et al. The learning curve for a surgeon in robot-assisted laparoscopic pancreaticoduodenectomy: a retrospective study in a high-volume pancreatic center. Surg Endosc. 2019;33(9):2927-33.

73. Shi Y, Wang W, Qiu W, et al. Learning curve from 450 cases of robot-assisted pancreaticoduocectomy in a high-volume pancreatic center: optimization of operative procedureand a retrospective study. Ann Surg. 2019; https://doi.org/10. 1097/SLA.0000000000003664.

74. Jones LR, Zwart MJW, Molenaar IQ, et al. Robotic pancreatoduodenectomy: patient selection, volume criteria, and training programs. Scand J Surg. 2020;109(1):29-33.

75. Kang CM, Kim DH, Lee WJ, et al. Conventional laparoscopic and robot-assisted spleen-preserving pancreatectomy: does da Vinci have clinical advantages? Surg Endosc. 2011;25(6):2004-9.

76. Guerrini GP, Lauretta A, Belluco C, et al. Robotic versus laparoscopic distal pancreatectomy: an up-to-date metaanalysis. BMCSurg. 2017;17(1):105.

77. Higgins RM, Frelich MJ, Bosler ME, et al. Cost analysis of robotic versus laparoscopic general surgery procedures. SurgEndosc. 2017;31(1):185-92.

78. van Hilst J, Korrel M, de Rooij T, et al. Oncologic outcomes of minimally invasive versus open distal pancreatectomy for pancreatic ductal adenocarcinoma: a systematic review and meta-analysis. Eur JSurg Oncol. 2019;45(5):719-27.

79. Min S, Kim N, Zhan G. The impact of market size on new market entry: a contingency approach. Eur J Mark. 2017;51:2-22.

80. Syverson C. Prices, spatial competition and heterogeneous producers: an empirical test. J Ind Econ. 2007;55(2):197-222. https://doi.org/10.1111/j.1467-6451. 2007.00308.x.

Publisher's Note Springer Nature remains neutral with regard to jurisdictional claims in published maps and institutional affiliations. 